com/syringe-driver-database-introduction.html. Zugegriffen:3.Nov. 2016

14. Negro $S$, Reyes R, Azuara ML, Sánchez Y, Barcia E (2006) Morphine, Haloperidol and hyoscine $\mathrm{N}$-butylbromide combined in s.c. infusion solutions: compatibility and stability. Int J Pharm 307(2):278-284

15. Bausewein C, Roller S, Voltz R (2015) Leitfaden Palliative Care, 5. Aufl. Urban \& Fischer Elsevier, München

16. Watson M, Lucas C, Hoy A, Back I (2005) Oxford handbook of palliative care, 1. Aufl. Oxford University Press, Oxford

17. Bruera E, MacEachern T, Macmillan K, Miller MJ, Hanson J (1993) Local tolerance to subcutaneous infusions of high concentrations of hydromorphone: A prospective study. J Pain Symptom Manage 8(4):201-204

18. Bausewein C (2007) Unter welchen Bedingungen ist die subkutane Gabe von Flüssigkeiten indiziert? Internist (Berl) 48(4):439-441

19. Herndon C (2001) Continuous subcutaneous infusion practices of United States hospices. J Pain Symptom Manage 22(6):1027-1034

20. Mitten T (2001) Subcutaneous drug infusions: a review of problems and solutions. Int J Palliat Nurs 7(2):75-85

21. Eisenchals JH et al (2005) Low-dose levomepromazine in refractory emesis in advanced cancer patients: an open-label study. Palliat Med 19(1):71-75

22. Forbat $L$ et al (2017) How and why are subcutaneous fluids administered in an advanced illness population: a systematic review. J Clin Nurs 26(9-10):1204-1216

\title{
Erratum
}

Onkologe $2017 \cdot 23: 664-665$

DOI 10.1007/s00761-017-0268-9

Online publiziert: 17. Juli 2017

๑) Springer Medizin Verlag GmbH 2017

CrossMark

Thomas Ettrich ${ }^{1}$ Stefan Schönsteiner ${ }^{2} \cdot$ Regine Mayer-Steinacker ${ }^{2}$. Hartmut Döhner ${ }^{2} \cdot{\text { Christiane } \text { Gog }^{3}}^{*}$ Peter Thuss-Patience ${ }^{4}$. Carolin Wolf ${ }^{5}$. Christoph Ostgathe ${ }^{6}$

${ }^{1}$ Klinik für Gastroenterologie, Endokrinologie, Stoffwechsel, Ernährungswissenschaften und Nephrologie, Comprehensive Cancer Center Ulm - Integratives Tumorzentrumdes Universitätsklinikums und der Medizinischen Fakultät, Ulm, Deutschland

${ }^{2}$ Klinik für Innere Medizin/Hämatologie und Internistische Onkologie/Palliativmedizin, Comprehensive Cancer Center Ulm - Integratives Tumorzentrum des Universitätsklinikums und der Medizinischen Fakultät, Ulm, Deutschland

${ }^{3}$ Palliativmedizinische Abteilung, Universitäres Centrum für Tumorerkrankungen Frankfurt, Universitätsklinikum Frankfurt der JohannWolfgang Goethe-Universität, Frankfurt am Main, Deutschland

${ }^{4}$ Zentrumfür Palliativmedizin,Medizinische Klinikmit Schwerpunkt Hämatologie, Onkologie und Tumorimmunologie, Comprehensive Cancer Center, Charité, Universitätsmedizin Berlin, Berlin, Deutschland

${ }^{5}$ Apotheke, Comprehensive Cancer Center CCC Erlangen-EMN, Universitätsklinikum Erlangen, FriedrichAlexander-Universität Erlangen-Nürnberg, Erlangen, Deutschland

${ }^{6}$ Palliativmedizinische Abteilung, Comprehensive Cancer Center CCC Erlangen-EMN, Universitätsklinikum Erlangen, Friedrich-Alexander-Universität Erlangen-Nürnberg, Erlangen, Deutschland

\section{Erratum zu: SOP - Darmpassagestörung in der Palliativmedizin}

\author{
Erratum zu: \\ Onkologe 2017 \\ DOI 10.1007/s00761-017-0239-1
}

In $\bullet$ Abb. 2 der Originalversion fehlt die therapeutische Handlung „systematische medikamentöse Tumortherapie" rechts oben.

\section{Korrespondenzadresse}

Dr. med. S. Schönsteiner, M.Sc.

Klinik für Innere Medizin/Hämatologie und Internistische Onkologie/Palliativmedizin, Comprehensive Cancer Center Ulm - Integratives Tumorzentrum des Universitätsklinikums und der Medizinischen Fakultät Ulm, Deutschland rebecca.sommer@uniklinik-ulm.de
Die Online-Version des Originalartikels ist unter DOI 10.1007/s00761-017-0239-1 zu finden. 


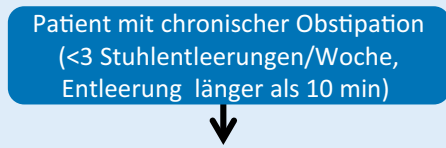

Angemessene diagnostische Maßnahmen durchführen

- Labor: Kalium, Kalzium

- Anamnese/körperliche Untersuchung:

Medikamente, Stuhlfrequenz, Konsistenz, Dauer der Entleerung, Stuhlmenge, Schmerzen, Defäkation, tastbare Kotballen, Darmgeräusche, rektale Austastung

- Technische Untersuchung: CT des Abdomens, Sonografie, Abdomenübersicht, Magen-Darm-Passage

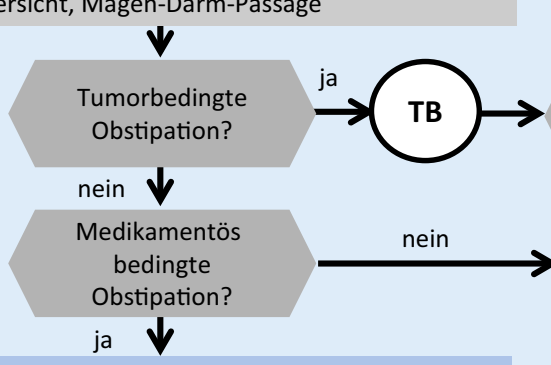

Weglassen der auslösenden Substanzen und Stufenschema bis

jeden 2./3. Tag ein weich, geformter Stuhl

1. z.B. Macrogol oder Bisacodyl oder Picosulfat

2. Kombination aus 2 Medikamenten der Stufe 1 z.B. Macrogol und Bisacody

3a. + Mannit oder Macrogol + rektale Maßnahmen (Klysma, Hebe-Senk-Einlauf, Bisacodyl supp.) fakultativ bei Opioidgabe

3b. bei Therapierefraktärität und opioidinduzierter Obstipation: Methylnaltrexoniumbromid

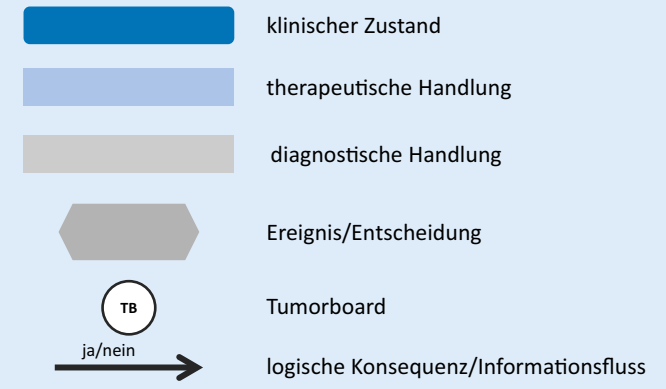

Palliativmedizinisches Gesamtkonzept

- Best palliative/supportive care an die Bedürfnisse des Patienten angepasst

Systematische medikamentöse Tumortherapie

Chirurgische Tumortherapie

Patient in die

Therapie ein?

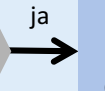
durchführen

\section{Situation- und individuellem Risiko}

entsprechend:

- Flüssigkeitszufuhr überprüfen und ggf. auf 1,5-2l steigern

- Körperliche Aktivität steigern

- Absetzen aller unnötig auslösenden Medikamente

- Ungestörtes Milieu

- Ballaststoffreiche Nahrung nur bei genügender Flüssigkeitszufuhr von 1,5-2। pro Tag

Abb. 2 - Diagnostik und Therapie der chronischen Obstipation 